\title{
Caracterización mediante la técnica EBSD de la deformación de chapa de acero inoxidable AISI 304 DDQ bajo tensiones multiaxiales típicas de la embutición ${ }^{(\cdot)}$
}

\author{
J. Coello*, V. Miguel*, A. Calatayud*, C. Ferrer** y A. Martínez*
}

Resumen

\begin{abstract}
El objetivo de este trabajo es evaluar el comportamiento del acero inoxidable AISI 304 DDQ durante un proceso de deformación típico del conformado de chapa por embutición, tracción-compresión biaxial (T-CC), determinando la evolución microestructural mediante microscopía óptica y EBSD. Se ha establecido la validez del análisis efectuado por EBSD para las condiciones de deformación consideradas en este trabajo. Se ha analizado la influencia de la dirección de laminación y de la deformación equivalente sobre los mapas de orientación cristalina, diagramas de desorientación y figuras de polos inversa, determinando que las desorientaciones de ángulo bajo obtenidas en muestras deformadas a $0^{\circ}, 45^{\circ}$, y $90^{\circ}$ respecto a la dirección de laminación del material presentan una correlación inversa con la anisotropía normal del material. También se ha estudiado la influencia del preestirado en condiciones de tensión uniaxial sobre las características microestructurales del material deformado en condiciones de tracción-compresión biaxial. Este estudio revela que el gradiente de desorientaciones intragranulares producidas en la deformación depende en gran medida de las condiciones iniciales del material; siendo dicho gradiente menor en las muestras con acritud inicial. El análisis de desorientaciones de ángulo alto $\left(>15^{\circ}\right)$ revela la existencia de la variación de la distribución del carácter del límite de grano con la deformación.
\end{abstract}

\section{Characterization by EBSD technique of AISI 304 DDQ stainless steel sheet deformed under typical deep drawing multiaxial stress system}

\begin{abstract}
The main aim of this work is to evaluate AISI 304 DDQ stainless steel behaviour under deep drawing deformation condition, that is, pure shear deformation in which material suffers a typical deformation under tension-biaxial compression stresses system. The microestructural evolution has been investigated by optical microscopy and by EBSD technique. The success of the EBSD analysis has been established for the deformation conditions experimented here. It has been determined the rolling direction and the equivalent strain influence on the crystallographic orientation maps, misorientation diagrams and poles figures. The results let the authors say the low angle misorientations corresponding to 0,45 and $90^{\circ}$ rolling directions have an inverse correlation with the material anisotropy. Initial prestraining has been considered also and the analysis of this aspects lead to establish that the increment of the intragranular misorientations with the strain depends on the initial state of the steel; this increment is observed to be minor for samples with initial prestraining. High angle misorientation analysis $\left(>15^{\circ}\right)$ indicates that the grain boundaries character distributions depends on the deformation.
\end{abstract}

Keywords

Stainless steel; Deep drawing; EBSD; Anisotropy.

\section{INTRODUCCIÓN}

Uno de los cambios importantes que puede sufrir un material policristalino durante un proceso de conformado es la formación de nuevas texturas (orientación preferente de los granos en determinadas direcciones cristalográficas). El estudio de texturas está basado en la determinación de direcciones preferenciales de orientación de forma cualitativa y el cálculo de la fracción de volumen asociada a cada una de esas orientaciones de forma cuantitativa. Dado que muchas propiedades de los materiales son

(•) Trabajo recibido el día 9 de octubre de 2008 y aceptado en su forma final el día 29 de enero de 2009.

* Instituto de Desarrollo Regional; Universidad de Castilla-La Mancha; Avda. España s/n 02006 Albacete.

** Departamento de Ingeniería Mecánica y de Materiales; Universidad Politécnica de Valencia; Camí de Vera s/n 46022 Valencia. 
anisótropas, el estudio de la textura tiene numerosas aplicaciones, entre las que destaca el control de los procesos de embutición ${ }^{[1]}$.

Tradicionalmente, la determinación de texturas se ha realizado mediante técnicas macroscópicas, como la difracción de rayos X y difracción de neutrones. Ambas técnicas permiten estudiar la orientación cristalina de un elevado número de granos y obtener una descripción estadística de la macrotextura. Sin embargo, actualmente, la técnica EBSD, basada en la difracción de electrones retrodispersados, es considerada como la más utilizada para el estudio de microtexturas y consiste en la determinación de las orientaciones individuales de un número significativo de granos cristalinos. Esta técnica, basada en los diagramas de Kikuchi ${ }^{[2 \mathrm{y} 3]}$, permite realizar, al mismo tiempo, un amplio análisis microestructural, el estudio de las orientaciones cristalinas, el análisis de fases existentes y la evolución de la microtextura, durante el proceso de deformación. En consecuencia, la técnica EBSD permite determinar el comportamiento del material durante el proceso de conformado del mismo, tal y como establecen diversos autores en procesos de recristalización dinámica típica de procesos de deformación en caliente ${ }^{[4-7]}$.

Otros autores, estudian las características microestructurales y las desorientaciones intergranulares e intragranulares producidas en diferentes procesos de deformación en frío. Xue et al. ${ }^{[8-11]}$ estudian mediante EBSD la evolución microestructural de muestras de acero inoxidable sometidas a esfuerzos de compresión por cizalladura en las proximidades a la zona fuertemente deformada. Este estudio revela que el acero AISI 304, tras el proceso de deformación, presenta una variación de textura en las zonas próximas a las bandas de deformación obteniéndose, generalmente, una reorientación de la dirección (110) en la dirección de cizalladura, y/o de los planos $\{111\}$ paralelos al del des-

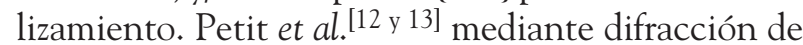
Rayos X y EBSD determinan la textura y evolución microestructural del acero austenítico 304 deformado mediante ensayos de tensión uniaxial a $-60^{\circ} \mathrm{C}$. Sus estudios demuestran que la martensita inducida por deformación, $\alpha^{\prime}$, está asociada con las bandas $\{111\}$.

No obstante, no existen estudios realizados que relacionen la evolución de la textura del acero en procesos de deformación típica del conformado de chapa por embutición, en los que la deformación característica en la zona del prensachapas se puede definir como planos de cizalladura, pure shear, inducida por esfuerzos de tracción-compresión biaxial (T-CC) ${ }^{[14]}$.

En los procesos de deformación en frío de aceros inoxidables austeníticos, la posible evolución de la textura del material va acompañada de una modificación microestructural por la aparición de fases inducidas con la deformación, esto es, martensita $\alpha^{\prime}$. Esto, hace de la técnica EBSD un excelente medio para el análisis correspondiente. Además, esta técnica permite analizar la textura del material a partir de la microtextura del mismo y, en particular, permite caracterizar la distribución de juntas de grano ${ }^{[2]}$.

En este trabajo se ha evaluado el comportamiento del acero 304 DDQ durante el proceso de embutición, estudiando la evolución microestructural de dichas muestras mediante microscopía óptica y EBSD. Se ha estudiado la influencia de la dirección de laminación y de la deformación transversal sobre los mapas de orientación cristalina, diagramas de desorientación y figuras de polos. También, se ha estudiado la influencia de una deformación previa en condiciones de tensión uniaxial aplicada al 304 DDQ, sobre las características microestructurales del material deformado únicamente en condiciones de traccióncompresión biaxial (T-CC). La evolución de la textura del material permitirá determinar el comportamiento del material durante la deformación, relacionando la influencia de la anisotropía del mismo y la variación que experimenta con la deformación ${ }^{[15]}$.

\section{DESARROLLO EXPERIMENTAL}

\subsection{Material utilizado}

Se ha empleado chapa de acero inoxidable austenítico AISI 304 DDQ de espesor 0,8 mm laminada en frío con un acabado superficial típico comercial 2B. Dicho acabado se obtiene mediante un proceso de recocido más decapado posterior a la laminación de la chapa ${ }^{[16]}$. La composición química y las propiedades mecánicas del acero se indican en las tablas I y II.

\subsection{Proceso de deformación bajo condiciones de tracción- compresión biaxial}

Con objeto de simular las condiciones de deformación que tienen lugar en un elemento del flanco duante el

\section{Tabla I. Composición química (\%) del acero AISI 304 DDQ}

Table I. AISI 304 DDQ steel chemical composition (\%)

\begin{tabular}{ccccccccc}
\hline $\mathbf{C}$ & $\mathbf{S}$ & $\mathrm{Si}$ & $\mathbf{M n}$ & $\mathrm{Cr}$ & $\mathrm{Ni}$ & $\mathrm{Mo}$ & $\mathbf{V}$ & $\mathbf{C u}$ \\
\hline 0,040 & 0,003 & 0,19 & 1,11 & 17,67 & 9,01 & 0,28 & 0,08 & 0,26 \\
\hline
\end{tabular}


Tabla II. Propiedades mecánicas y plásticas del acero AISI 304 DDQ ( $r$ y n corresponden a los índices de anisotropía normal y al de endurecimiento, respectivamente)

Tabla II. Mechanical and plasticity properties of AISI $304 D D Q$ steel ( $r$ and $n$ are normal anisotropy and strengthening coefficients, respectively)

\begin{tabular}{cccccc}
\hline Acero & $\begin{array}{c}\mathbf{R}_{\mathrm{p} 0.2} \\
(\mathrm{MPa})\end{array}$ & $\begin{array}{c}\mathrm{Rm} \\
(\mathrm{MPa})\end{array}$ & $\mathrm{HV} 0,5$ & $\mathbf{r}$ & $\mathbf{n}$ \\
\hline $304 \mathrm{DDQ}(2 \mathrm{~B})$ & 252 & 582 & 174 & 0,906 & 0,244 \\
\hline
\end{tabular}

proceso de embutición profunda de chapa se ha realizado un ensayo de deformación en condiciones de tracción-compresión biaxial. Con anterioridad, el ensayo ha sido definido y validado como representativo del proceso $^{[17-18]}$. Este ensayo está basado en el estirado de probetas planas empleando un conjunto matriz-pisador en forma de cuña con un ángulo de $30^{\circ}$. Mediante un sistema de cierre se aplica una fuerza normal al conjunto matriz-pisador, entre los que se aloja la muestra de ensayo. Con la aplicación de una tensión de estirado se hace pasar el material por la matriz, que experimenta una deformación plana debido a la compresión lateral de la chapa (Fig. 1).

Las probetas ensayadas se obtuvieron por corte, mediante cizalla de tiras de chapa con una anchura inicial constante, $h_{0}$, variable en el rango de 11,5 a $15,5 \mathrm{~mm}$. Se seleccionaron muestras en la dirección de $0^{\circ}, 45^{\circ}$ y $90^{\circ}$, con respecto a la de laminación. Para poder introducir las muestras en la matriz, se

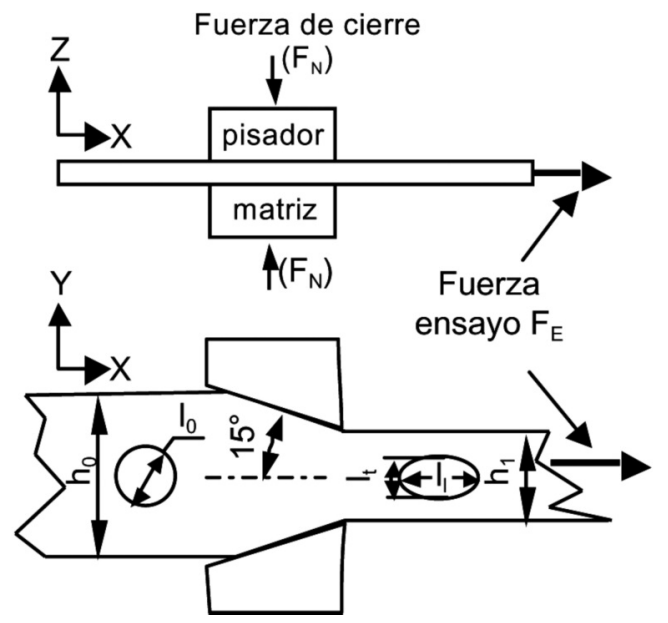

mecanizó un estrangulamiento con la forma de la matriz mediante una muela de esmeril.

Para evaluar las deformaciones producidas en el material tras el ensayo, se grabaron de forma mecánica circunferencias de diámetro conocido, $1_{0}$. Las deformaciones longitudinal, $\varepsilon_{\mathrm{l}}$, y transversal, $\varepsilon_{\mathrm{t}}$, producidas tras el ensayo pueden evaluarse conforme a las ecuaciones (1) y (2), teniendo en cuenta las dimensiones finales de las circunferencias en el sentido del estirado, $1_{1}$, y en el transvesal, $1_{t}$, respectivamente.

$$
\varepsilon_{\mathrm{I}}=\ln \left(\frac{\mathrm{I}_{\mathrm{I}}}{\mathrm{I}_{0}}\right)
$$

$$
\varepsilon_{\mathrm{t}}=\ln \left(\frac{\mathrm{I}_{\mathrm{t}}}{\mathrm{I}_{0}}\right)
$$

La deformación en el sentido del espesor de la chapa, $\varepsilon_{z}$, se obtiene a partir de $\varepsilon_{1}$ y $\varepsilon_{\mathrm{t}}$, considerando la hipótesis de volumen constante, conforme a la ecuación (3).

$$
\varepsilon_{z}=\varepsilon_{l}-\left|\varepsilon_{t}\right|
$$

Finalmente, la deformación equivalente de Von Misses se obtiene conforme a la expresión (4).

$$
\varepsilon_{e q}=\sqrt{\frac{2}{3}\left(\varepsilon_{l}^{2}+\varepsilon_{t}^{2}+\varepsilon_{z}^{2}\right)}
$$

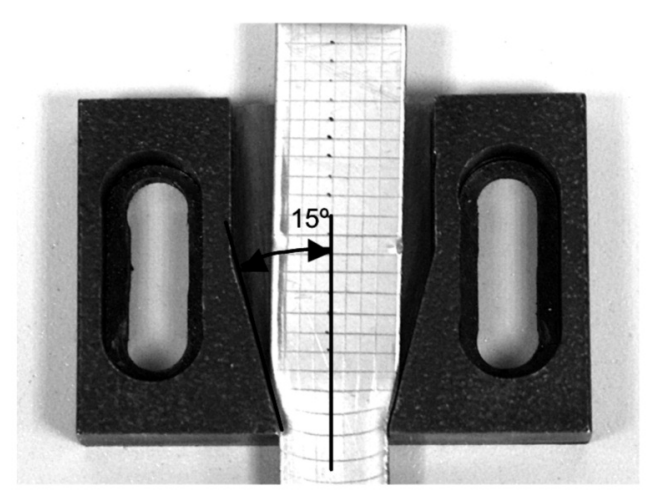

Figura 1. Fundamento del ensayo tracción-compresión biaxial (T-CC). Detalle de la matriz de ensayo.

Figure 1. Tensile-biaxial compression test fundamentals (T-CC). Test die detail. 
El rango de deformación equivalente aplicada en los ensayos ha sido entre 0,1 y 0,33 , en las condiciones indicadas de tracción-compresión biaxial. La deformación equivalente ejecutada en los ensayos de preestirado bajo condiciones de tensión uniaxial fue 0,065 y 0,18 .

\subsection{Caracterización de las muestras deformadas}

Las muestras ensayadas con diferentes grados de deformación se han caracterizado mediante microscopía óptica y mediante la técnica SEM-EBSD desarrollada en un microscopio electrónico de barrido Jeol JSM -7200 de filamento de Wolframio, equipado de un detector EBSD HKL Technology.

El estudio de microscopía óptica se ha realizado sobre una sección transversal de las diferentes muestras deformadas en condiciones T-CC, con y sin acritud inicial. Sobre cada una de estas muestras se ha realizado un barrido de microdurezas $\mathrm{HV} 0,5$, desde la zona del borde hasta el centro de las mismas.

El análisis EBSD se ha llevado a cabo sobre una sección superficial $(50 \times 50 \mu \mathrm{m})$ extraída de la parte central de las distintas muestras deformadas, que presenta una deformación uniforme. La preparación de las muestras se ha realizado mediante desbaste y pulido mecánico con pasta de diamante de 7,3 y 1 $\mu \mathrm{m}$. Para eliminar la capa superficial deformada como consecuencia de la preparación indicada, las muestras, finalmente, son pulidas con alúmina y atacadas ligeramente con una solución de $10 \%$ $\mathrm{HNO}_{3}$ y $15 \% \mathrm{HF}$ en agua. Sobre cada una de estas muestras se han realizado dos barridos de $250 \mathrm{x}$ $190 \mu \mathrm{m}^{2}$ con un tamaño de paso de $0,48 \mu \mathrm{m}$, considerando la dirección de embutición como la rolling direction (RD) en el sistema de referencia SEMEBSD. Se ha considerado, como criterio de tolerancia para la definición de grano, un cambio de orientación de $5^{\circ}$. Mediante los mapas e histogramas de desorientación se ha realizado el estudio de la distribución del carácter de los límites de grano y de las CSLs o juntas de grano especiales (coincidence sites lattices) determinando la densidad de desorientaciones de ángulo bajo $\left(3-15^{\circ}\right)$, de ángulo alto $\left(15-55^{\circ}\right)$, de maclas y de CSLs. La densidad de desorientaciones, IMD, se ha definido a partir de la ecuación (5), en función de las desorientaciones mínimas y máximas consideradas, $\phi_{\mathrm{O}}$ y $\phi_{\max }$ respectivamente, del número de pixels en el área escaneada, N, y del número de desorientaciones correspondientes a un ángulo $\phi$ de desorientación expresado en radianes, $\mathrm{MD}(\phi)$.

$$
I M D_{\phi}=\frac{\sum_{\phi_{0}}^{\phi_{\max }} \phi \cdot M D(\phi)}{N}
$$

En definitiva, IMD representa la función de distribución de las desorientaciones para un determinado ángulo, $\phi=\phi_{\max }$, cuya función de densidad es $\operatorname{MD}(\phi)$.

Estas desorientaciones también se expresan mediante la ecuación (6), en la que $\mathrm{n}_{\phi:}$ es el número de desorientaciones existentes de ángulo $\phi$.

$$
\%_{\phi}=\frac{n_{\phi}}{N}
$$

\section{RESULTADOS Y DISCUSIÓN}

\subsection{Caracterización de la deformación producida en condiciones de tracción-compresión biaxial}

El estudio microestructural, realizado sobre una sección transversal de las muestras embutidas con distinto grado de deformación, revela que la deformación producida no es totalmente uniforme en toda la probeta, pudiéndose observar tres zonas claramente diferenciadas como consecuencia del efecto de contorno que imponen los laterales de la matriz sobre la muestra de ensayo ${ }^{[19]}$. El extremo de la muestra (Fig. 2 a)), presenta una gran deformación como consecuencia de las elevadas tensiones de cizalladura existentes. Esta zona más afectada por el efecto lateral de la matriz tiene una extensión de aproximadamente $1 \mathrm{~mm}$. En la parte central, se observa una zona de aproximadamente $6 \mathrm{~mm}$, en la que la estructura es homogénea con una gran cantidad de líneas de deslizamiento y de maclas (Fig. 2 b)). La transición entre ambas partes se realiza de manera progresiva con una deformación no uniforme y decreciente hacia el centro de la muestra.

Se han efectuado barridos de microdureza Vickers sobre las muestras deformadas. En las figuras 3 y 4 se representan los valores de dureza en función de la distancia al borde de la muestra, obtenidos para distintos grados de deformación sin acritud previa o considerando ésta, respectivamente.

Se puede observar que la dureza es más elevada en la zona del borde, con una disminución brusca hasta una distancia de, aproximadamente, $2 \mathrm{~mm}$. A continuación, la dureza se mantiene prácticamente constante hasta el centro de la muestra. Este comportamiento, lógicamente, es coherente con las 

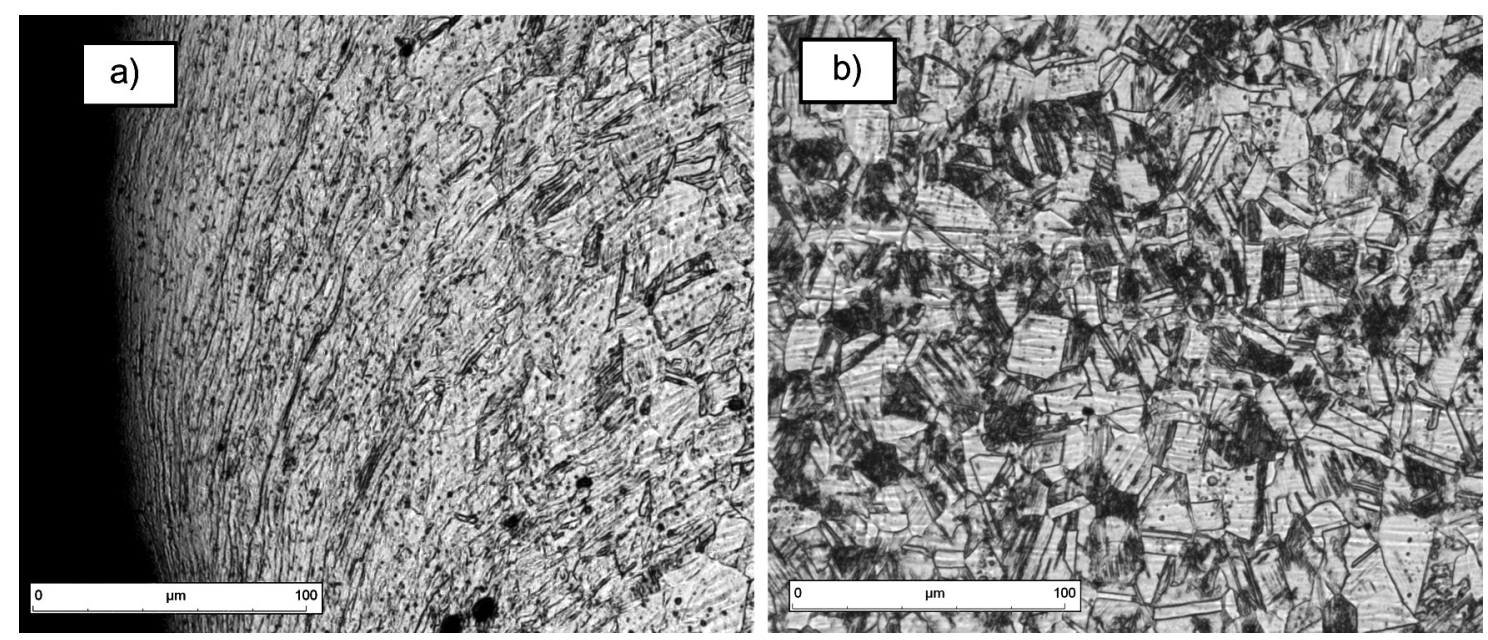

Figura 2. Definición microestructural de zonas en el material deformado en condiciones de T-CC. a) Micrografía de la zona del borde, en la que se observa el efecto de contorno provocado por la pared lateral de la matriz; b) Micrografía de la zona central, con deformación uniforme.

Figure 2. Different zones in the deformed material under T-CC conditions, based on optical microstructural analysis. a). Border area in which the die lateral wall effect can be observed; $b$ ). Central zone presents uniform deformation.

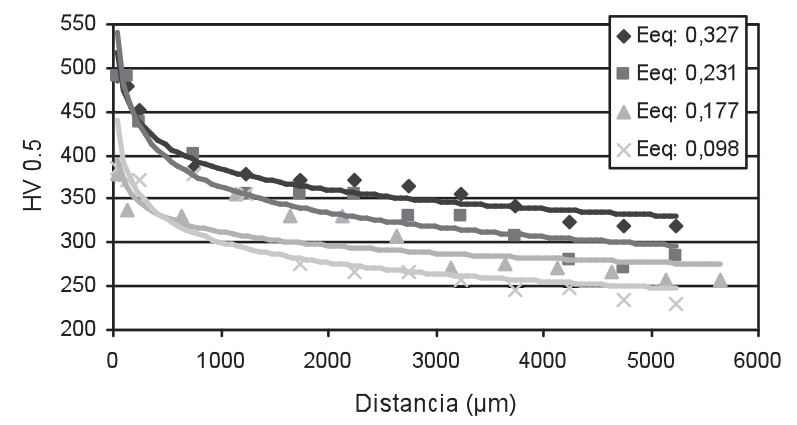

Figura 3. Perfiles de microdureza del acero AlSI $304 \mathrm{DDQ}$, estirado en condiciones de tracción-compresión biaxial con distintos grados de deformación. Ensayos realizados sin acritud inicial.

Figure 3. Microhardness profiles for AISI 304 $D D Q$ steel for different deformation grade under T-CC conditions. All assays are made without prestraining.

distintas zonas identificadas mediante microscopía óptica. Mayores valores de deformación conducen a durezas más elevadas, naturalmente.

Todo indica que el efecto de contorno en el ensayo se extiende a $2 \mathrm{~mm}$ a cada lado de la muestra pudiendo considerarse el resto como representativo de las condiciones de tracción-compresión biaxial que tienen lugar en los procesos de embutición de chapa.

\subsection{Influencia de la acritud inicial}

Para evaluar la influencia de la acritud inicial, obtenida por estirado, en el comportamiento del material frente a la deformación típica de los procesos de embutición, se han practicado dos grados de deformación equivalente mediante tracción uniaxial. Posteriormente, el material deformado se somete a las condiciones indicadas de tracción-compresión biaxial.

En la figura 4 se indican los valores de dureza obtenidos finalmente para dos grados de acritud inicial y dos deformaciones practicadas sobre el material, observándose que la acritud inicial sobre el material da lugar a un mayor endurecimiento final del mismo.

La figura 5 a) muestra los valores medios de dureza de la banda de deformación uniforme para las diferentes muestras procesadas en función de la deformación equivalente. Se observa que existe un ajuste lineal de la función lo que significa que la dureza está directamente relacionada con el grado de deformación equivalente. En la figura 5 b) se representan las pendientes de las rectas de dureza frente a la acritud inicial del material, determinándose también una relación directa entre éstas. En consecuencia, se puede establecer que la acritud inicial determina el gradiente del endurecimiento del material con la deformación con tendencia a un valor de dureza máximo independiente del grado de acritud inicial.

Finalmente, si se compara el endurecimiento adquirido por el material en distintas condiciones de deformación, esto es, comparando el proceso de 
Eeq: 0.187

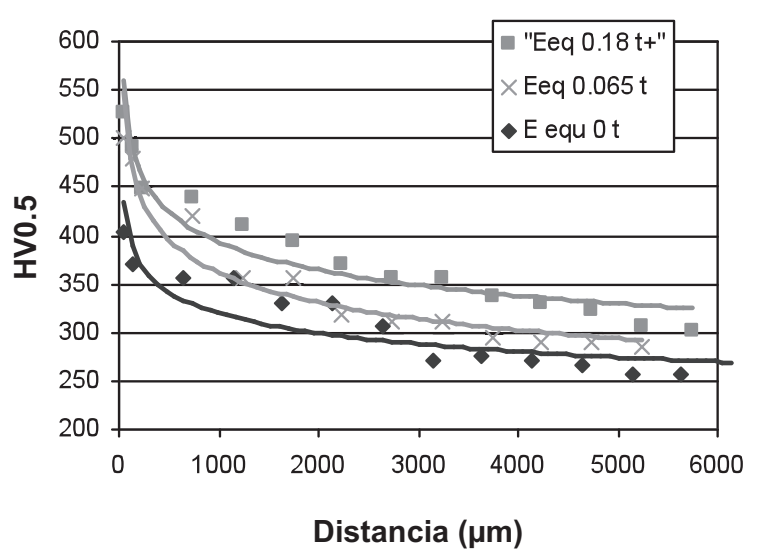

Eeq: 0.334

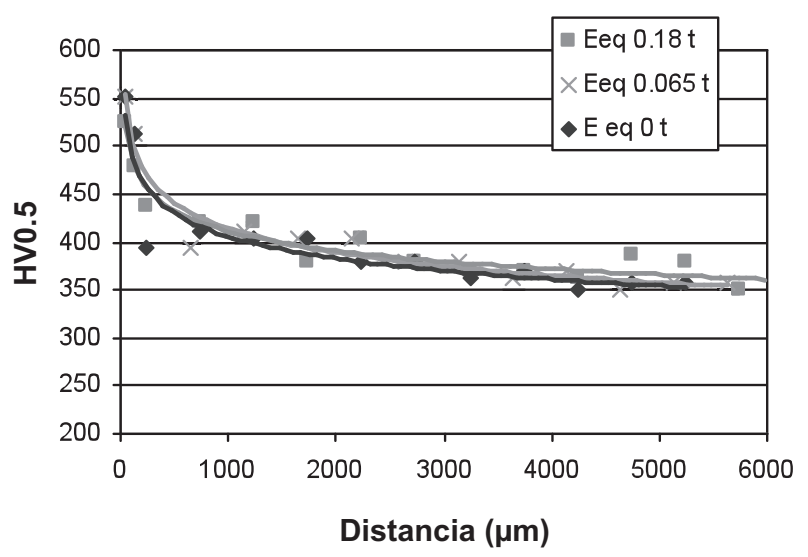

Figura 4. Perfiles de microdureza del acero AISI 304 DDQ deformado en condiciones de traccióncompresión biaxial con diferentes grados de acritud inicial.

Figure 4. Microhardness profiles for AISI 304 DDQ steel formed under T-CC conditions and for different uniaxial tensile prestraining grades.
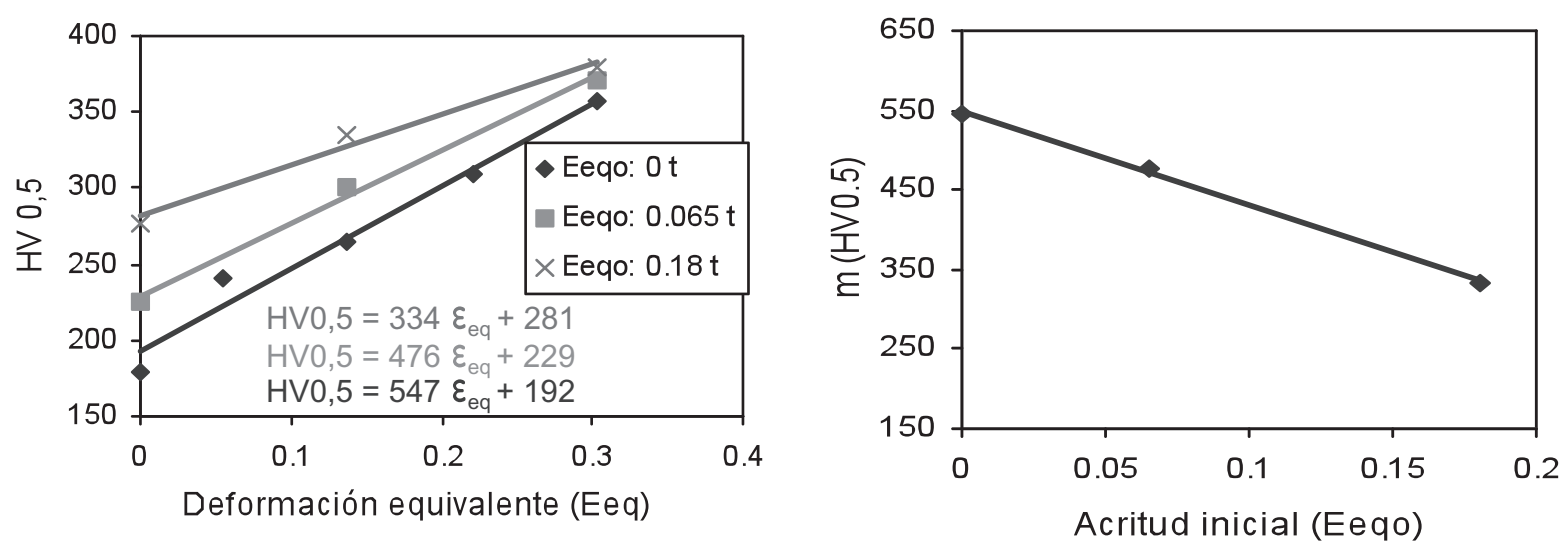

Figura 5. a) Dureza HV0.5 en función de la deformación equivalente, $\varepsilon_{\mathrm{q}}$, producida en condiciones de tracción-compresión biaxial; b) Pendientes de las rectas de dureza (5 a)) en función de la acritud inicial experimentada por el material.

Figure 5. a) HVO, 5 hardness as a function of equivalent Von Mises deformation, $\varepsilon_{q}$, under T-CC stresses condition; b) slopes of the hardness behaviour lines (5a) as a function of initial prestrain grade.

endurecimiento mediante tracción-compresión biaxial y mediante tracción uniaxial, se puede establecer que, para los valores de $\varepsilon_{\text {eq }}$ ensayados, no existe una influencia apreciable del proceso de deformación (Fig. 6).

\subsection{Caracterización de las muestras deformadas mediante EBSD}

Las muestras deformadas se han investigado selectivamente, en las diversas zonas de deformación indicadas con anterioridad, mediante la técnica EBSD.
La zona del material próxima al borde, fuertemente deformada, presenta problemas de identificación mediante EBSD debido a la baja intensidad de los diagramas de Kikuchi obtenidos y la imposibilidad de ser correctamente indexados. El porcentaje de área sin identificar en el mapping es muy elevada y, por tanto, la información de estos ensayos es muy poco precisa. En la figura 7 se representa el mapa de calidad y el mapa de fases correspondiente a la zona del borde, para una muestra con un $\varepsilon_{\text {eq }}$ de 0,327 sin acritud inicial. Las distintas fases aparecen representadas por colores, en la que el negro representa el porcentaje de muestra sin identificar $(51,6 \%)$. 


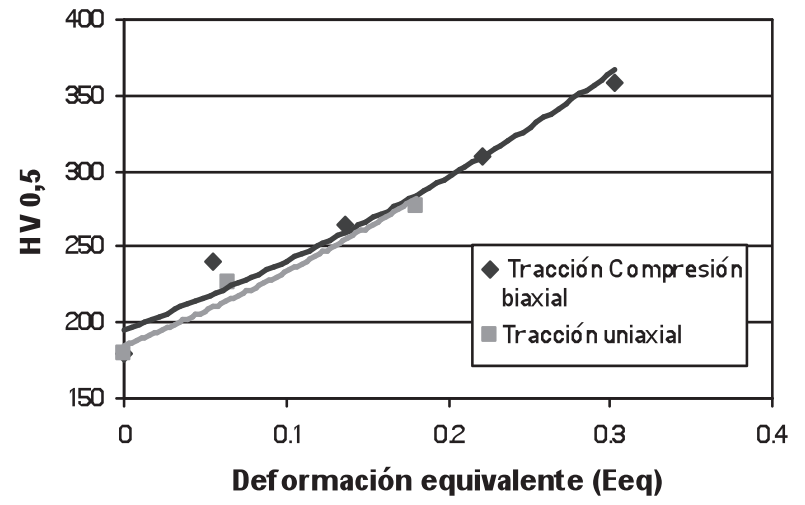

Figura 6. Valores de dureza HV 0,5 del material en función de la deformación equivalente, $\varepsilon_{\text {eq }}$ practicada por diferentes mecanismos de deformación.

Figure 6. HV 0,5 hardness values as a equivalent strain function, $\varepsilon_{e q}$, for different deformation systems.

Generalmente, los factores que disminuyen la calidad de los mapas obtenidos son: la presencia de subestructuras de tamaño inferior a la resolución del método, una alta densidad de dislocaciones debida a una elevada deformación y la preparación de las muestras $^{[2]}$. Particularmente, la presencia de martensita inducida por deformación, de tamaño nanométrico, formada en las intersecciones de maclas como zonas energéticamente favorables para ello, justifica la baja calidad del mapping en el caso del acero ensayado ${ }^{[9]}$, debido a que el tamaño de la fase inducida en la de- formación es inferior al tamaño de paso de barrido utilizado en el método. La presencia de subestructuras de pequeño tamaño, cuya formación está directamente relacionada con la cantidad de deformación efectuada, también actúa en el sentido indicado.

En la zona del centro de la probeta ensayada, de deformación uniforme, la calidad de los diagramas es muy superior ya que la deformación local es menor, tal y como se justifica mediante el análisis de dureza, con formación de fases inducidas y subestructuras de un tamaño superior a la resolución del método utilizado. Como consecuencia, puede establecerse la validez de la técnica y el método empleado para la caracterización de la evolución microestructural del material con la deformación, al ser esta zona del material la que representa las condiciones de deformación del proceso de embutición de chapa.

Por tanto, se puede investigar la evolución microestructural del acero inoxidable con la deformación, con base en el análisis de los mapas de orientación y la evolución de los límites de grano y de las subestructuras formadas en el interior de los granos.

En la figura 8 se muestran los mapas de orientación cristalina del material en estado de recepción y con diferentes grados de deformación. La diferente coloración está relacionada con la orientación cristalina característica de la estructura granular del material. Aunque cada grano se caracteriza por una coloración que define la orientación predominante, se pueden observar diferentes tonalidades que nos indican la presencia de distintas orientaciones dentro de un grano producidas por las numerosas dislocaciones y subestructuras producidas en la deformación ${ }^{[5]}$. Si se
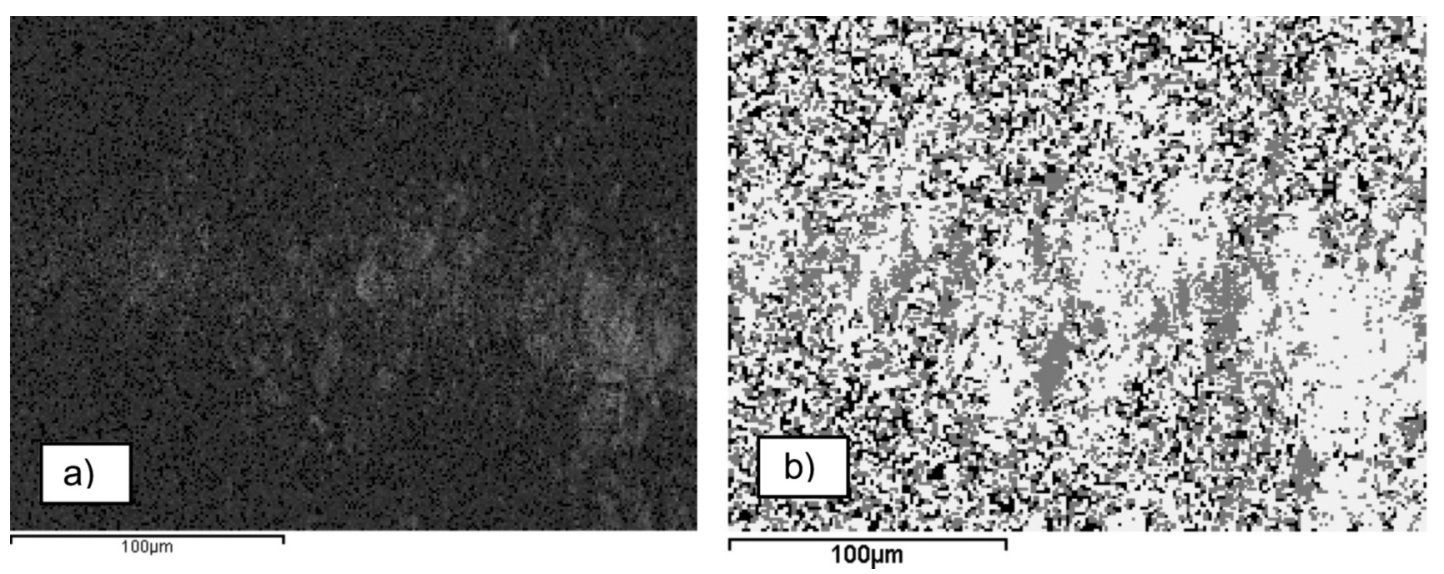

Figura 7. Muestra deformada en condiciones de tracción-compresión biaxial; $\varepsilon_{\mathrm{eq}}$ 0,337; Zona del borde. a) Mapa de calidad; b) Mapa de fases: austenita, $\gamma$ (claro) y martensita inducida por deformación, $\alpha^{\prime}$ (gris).

Figure 7. Forming sample under T-CC conditions; $\varepsilon_{\text {eq }} 0.337$; border zone. a) Image quality map; b) Phase map: austenite, $\gamma$ (clear) and strain induced martensite, $\alpha^{\prime}$ (grey). 

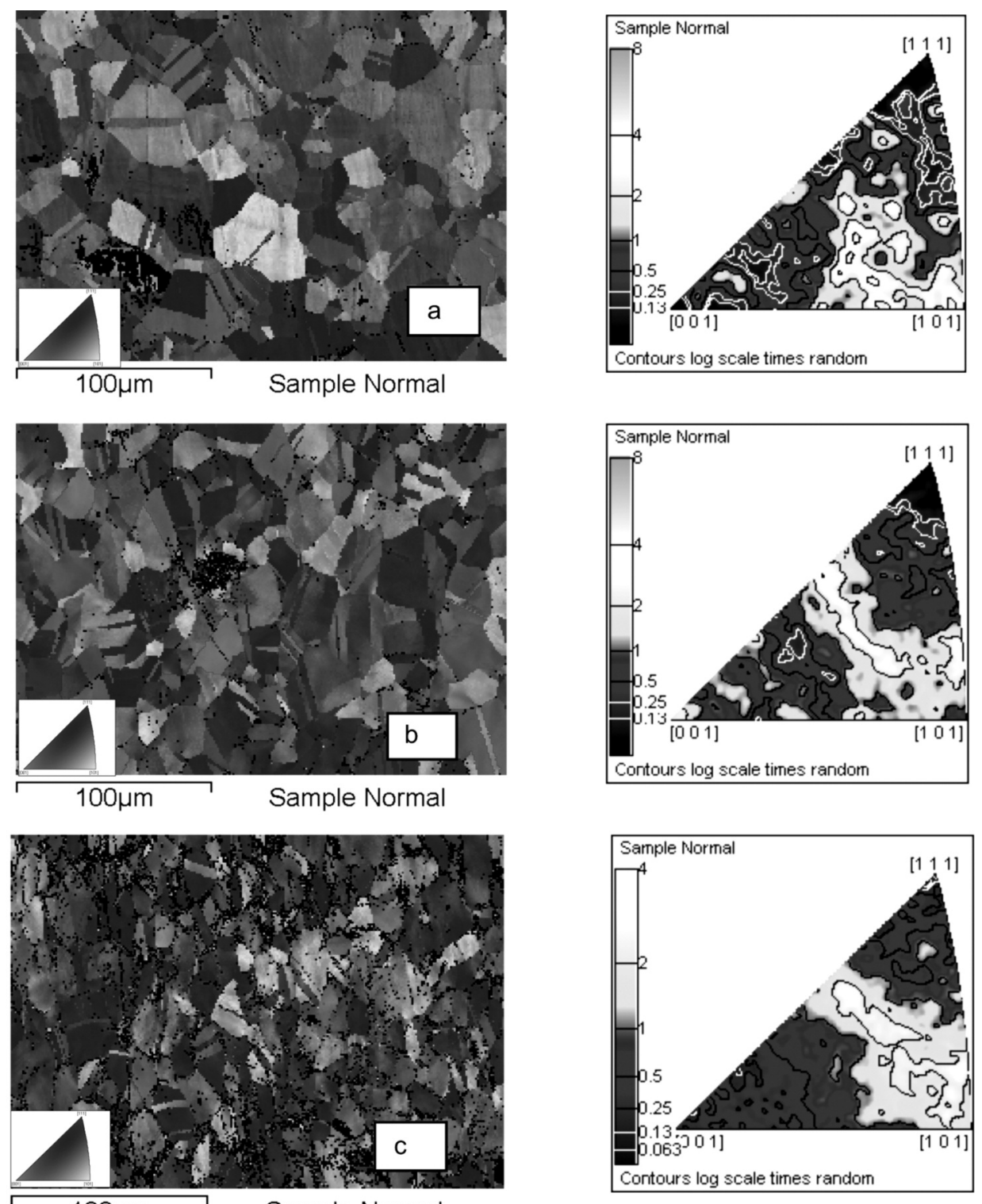

Figura 8. Mapas de orientación y figura de polos inversa del acero AISI 304 DDQ. a) En estado recepción, $\varepsilon_{\text {eq }} 0$; b) $\varepsilon_{\text {eq }} 0,177$; c) $\varepsilon_{\text {eq }} 0,327$.

Figure 8. Orientation maps and inverse pole figures for AISI 304 DDQ steel. a) Without any deformation, $\varepsilon_{\text {eq }} 0$; b) $\varepsilon_{\text {eq }} 0.177$; c) $\varepsilon_{\text {eq }} 0.327$.

observa la estructura del material en estado de recepción, esto es, recocido tras el proceso de laminación, se constata que los diferentes granos tienen una coloración bien definida y uniforme para todo el grano. En cambio, en el material deformado la gama de tonalidades dentro de cada grano es destacable, lo que indica la generación de las subestructuras mencionadas vinculadas al proceso de deformación sobre el material. Este cambio local de las orientaciones cristalinas producidas en el interior de los granos también es observado en las figuras de polos inversas mostradas en la figura 8 , en las que se observa una gran dispersión de orientaciones cristalinas que aumenta con la deformación. Esto, contrasta con la figura de polos inversa del material de partida en el que los puntos aparecen más agrupados, representando la orientación de los diferentes granos individuales que pueden ser observados claramente en el mapa de orientación y que presentan una mayor intensidad en el plano [101] con respecto a la dirección normal a la de estirado y, por tanto, en la dirección de la anchura de la muestra.

En la figura 9 se muestra el mapa y distribución de desorientaciones del material en estado de recepción, en la que se observan las desorientaciones de ángulo 

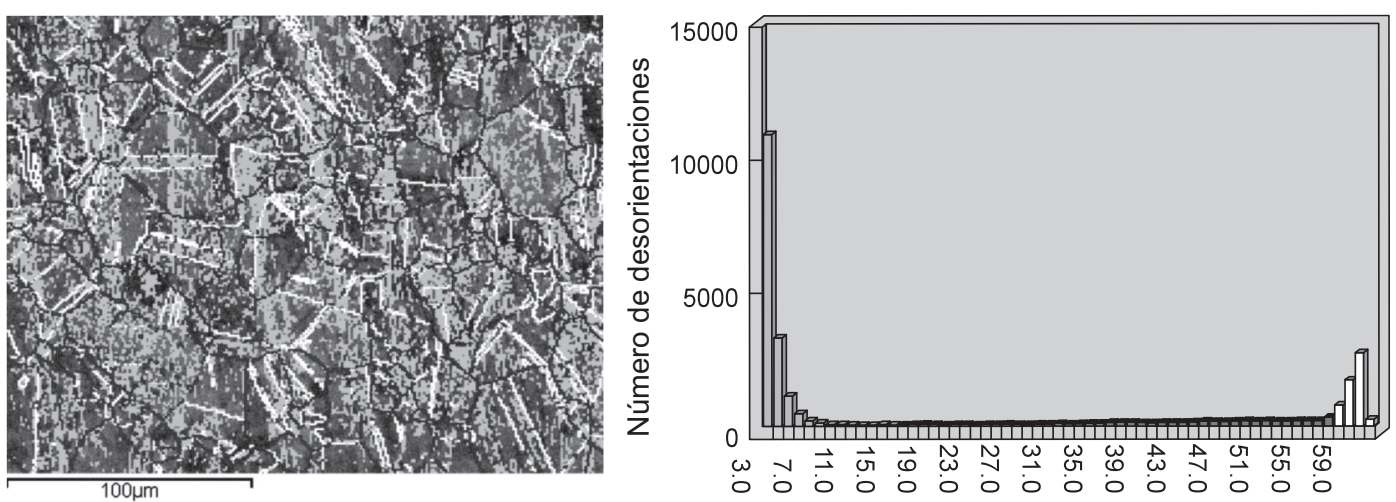

Angulo de desorientación $\left({ }^{\circ}\right)$

Figura 9. Mapa e histograma de desorientación del material en estado de recepción.

Figure 9. Orientation image mapping and misorientation distribution histogram for the material in initial conditions (without any deformation).

bajo en verde, las desorientaciones de ángulo alto en rojo (límites de grano) y las desorientaciones de $60^{\circ}$ en color blanco. Las desorientaciones de ángulo bajo, aunque pueden aparecer en materiales completamente recristalizados, también tienen correspondencia con los mecanismos de deformación intragranulares. Las desorientaciones de ángulo alto se corresponden con los fenómenos de deformación en límite de grano, si bien las desorientaciones a $60^{\circ}$ se pueden asociar no solo a juntas de grano sino también a maclas existentes en el material.

Para correlacionar las desorientaciones con la deformación aplicada, se han considerado dos parámetros: la densidad de desorientaciones, IMD, calculada (Ec. (6)). En la figura 10 aparece la relación existente entre estos dos parámetros y la deformación plástica producida en condiciones de tracción-compresión biaxial del material con y sin acritud inicial para las desorientaciones de ángulo bajo, maclas y ángulo alto. Aunque el número de puntos no es significativo, para bajas desorientaciones (Fig. 10 a)) se puede establecer una tendencia de tipo creciente con la deformación, lo que justifica que la densidad de desorientaciones de bajo ángulo, IMD (3-15을 y el \% de desorientaciones, son indicadores de la deformación practicada en el material. Si se comparan los resultados obtenidos con y sin preestirado por tensión uniaxial, se puede establecer que no existe correlación entre el preestirado y las desorientaciones de ángulo bajo en el rango de deformaciones practicado. En la figura $10 \mathrm{a}$ ) y b) se ha representado el IMD y el \% de las desorientaciones de ángulo alto, que aumentan con la deformación. La figura 10 c) muestra el IMD y el \% de desorientaciones en el rango de $57-60^{\circ}$. Se constata que ambos parámetros presentan una tendencia ligeramente decreciente con la deformación.
Los resultados indicados no se relacionan con el comportamiento mecánico observado para el material a partir de los ensayos de dureza. Efectivamente, aunque el gradiente de dureza con la deformación es menor para las muestras con acritud inicial, no se observa dicha tendencia en las desorientaciones producidas en la microestructura del material. También, este resultado difiere del obtenido por Xue et al. ${ }^{[8]}$ para muestras deformadas en caliente por tensiones de tipo cortante, en lo que podría considerarse como un efecto combinado de la deformación y de la recristalización dinámica.

Las desorientaciones de ángulo bajo existentes en el material de partida indican la presencia de efectos heredados de la textura de laminación de la chapa, a pesar del recocido que sufre el material con posterioridad a la misma. Si se comparan los resultados de las muestras con y sin acritud inicial no deformadas se constata un efecto de reorientación estructural en el material, como consecuencia del preestirado por tensión uniaxial, con tendencia a disminuir las desorientaciones de ángulo alto y de ángulo bajo.

En la figura 11 se representa la distribución de desorientaciones de ángulo bajo. Para cualquier grado de deformación, se observa que el número de desorientaciones disminuye muy rápidamente al principio, esto es, para los ángulos de desorientación más bajos. La tendencia a la disminución cambia significativamente para un rango de valores del ángulo de desorientación que es función de la deformación practicada. Así, para una deformación uniaxial de 0,065 el rango de valores se sitúa por debajo de 4. Para las otras deformaciones practicadas, dicho rango se sitúa entre $5^{\circ}$ y $8^{\circ}$, dependiendo de la deformación. Wilkinson et al. ${ }^{[20]}$ establecen que desorientaciones intragranulares inferiores a $6^{\circ}$ se corresponden con 

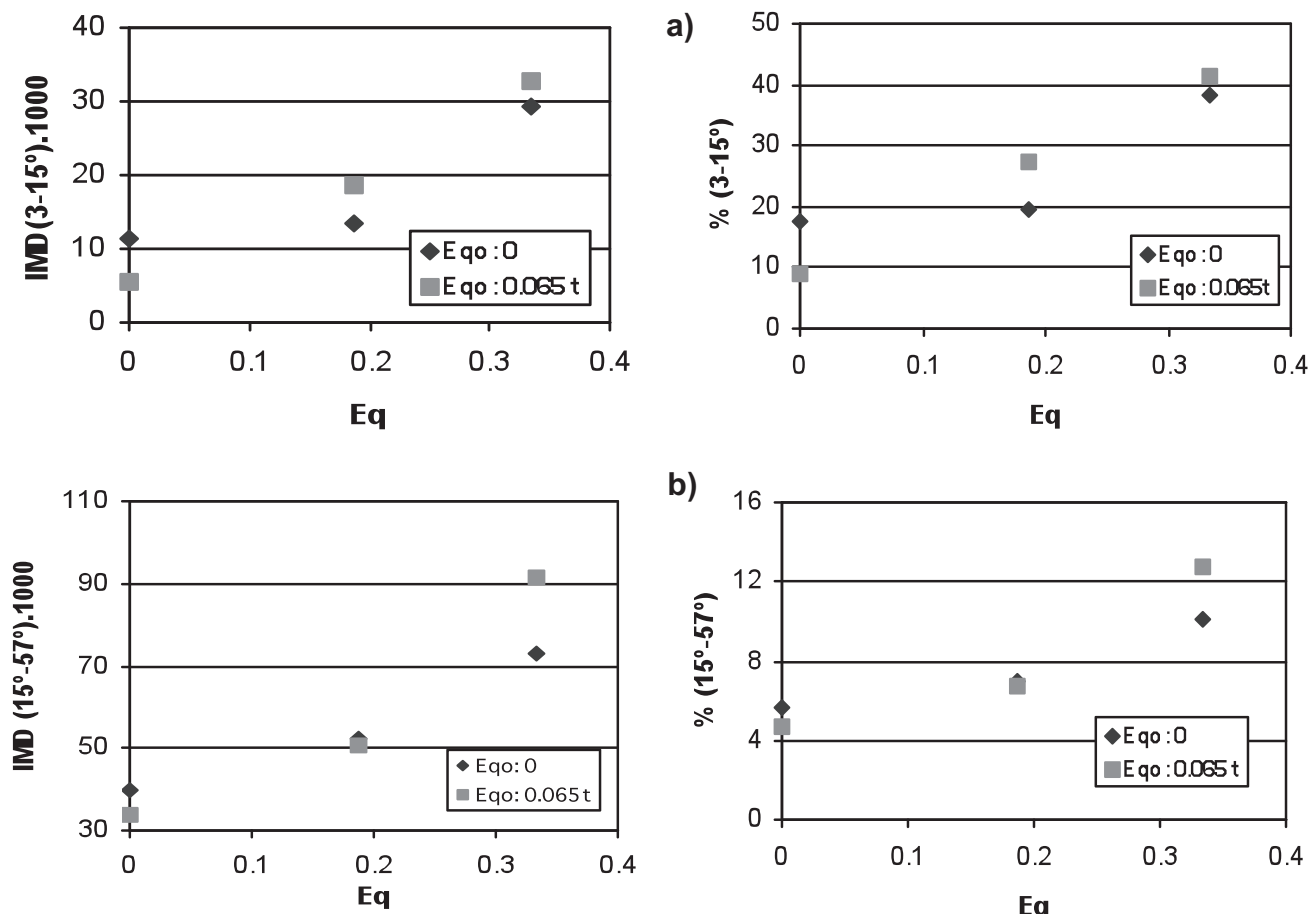

b)
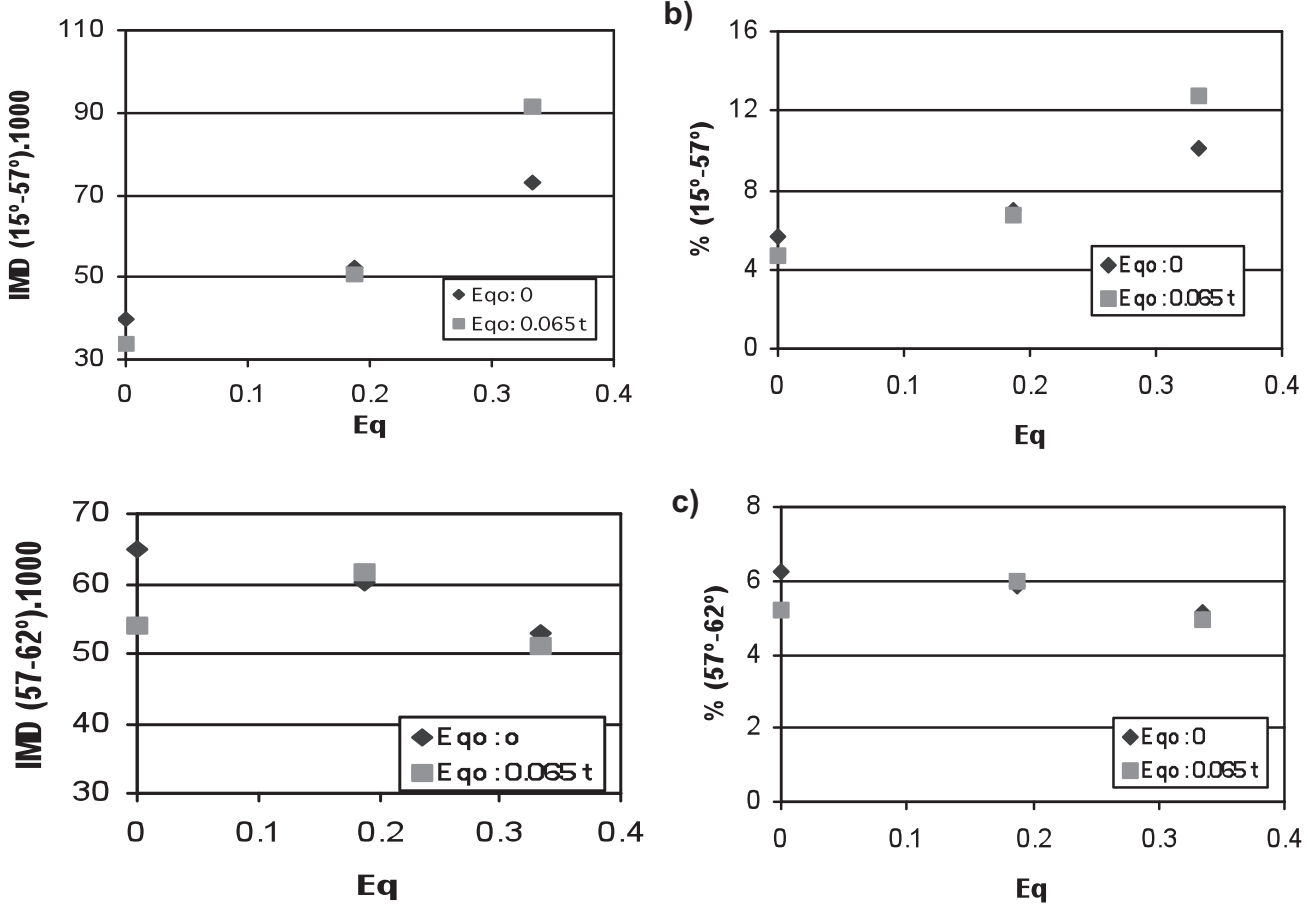

Figura 10. Variación de la densidad y del porcentaje de desorientaciones con la deformación equivalente: a) Desorientaciones de ángulo bajo; b) Desorientaciones de ángulo alto; c) Maclas.

Figure 10. Density and misorientations distributions variation as a function of equivalent strain; a) Low angle misorientations; b) High angle misorientations; c) Twins.

la formación de subgranos y subestructuras con distinta orientación. Si se adopta este valor como referencia para el análisis de los resultados mostrados en la figura 11, se constata que la deformación en condiciones T-CC afecta apreciablemente a la formación de las estructuras indicadas. A mayores grados de deformación la distribución de desorientaciones se desplaza hacia ángulos mayores.

\subsection{Influencia de la dirección de laminación}

Se ha evaluado, mediante EBSD, la influencia de la dirección de laminación con respecto a la dirección de estirado sobre las características microestructurales de las muestras deformadas. Si se comparan los diagramas de desorientación obtenidos en muestras deformadas a $0^{\circ}, 45^{\circ}$ y $90^{\circ}$ con respecto a la dirección de laminación (Fig. 12), se constata que sólo las desorientaciones de ángulo bajo presentan una correlación inversa con los valores de anisotropía normal, r. La correlación de las subestructuras, en términos de IMD, con la dirección de laminación se visualiza en la figura 12 d). En definitiva, se puede establecer que la dirección con menor tendencia al adelgazamiento del material en condiciones de embutición se corresponde con aquélla que presenta un menor número de desorientaciones después de haber sido deformada en un sistema de tensiones similar (T-CC). 


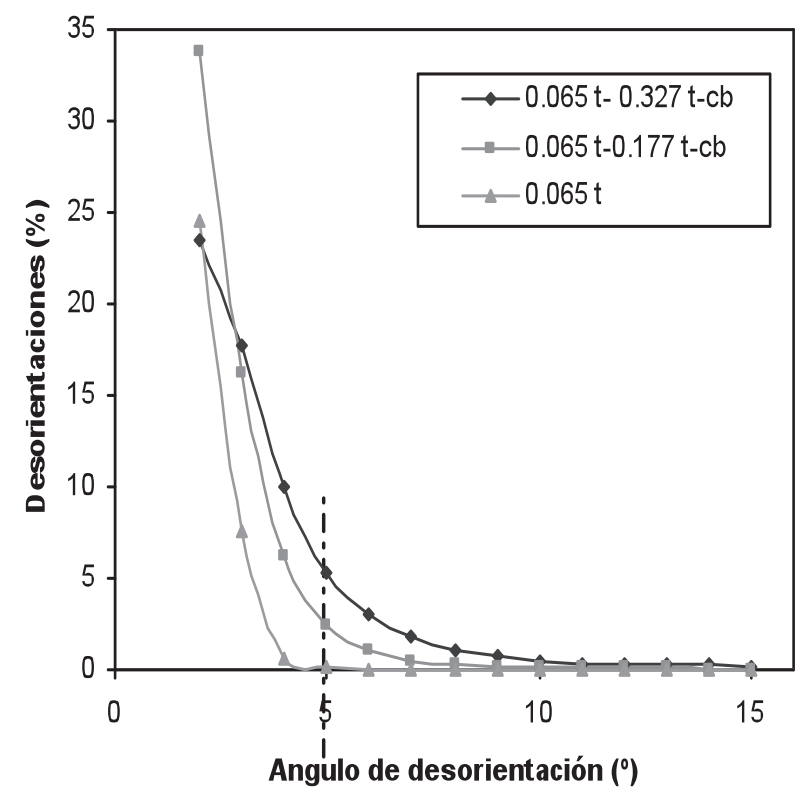

Figura 11. Desorientaciones de ángulo bajo para distintos grados de deformación.

Figure 11. Low angle misorientations for some deformation grades.
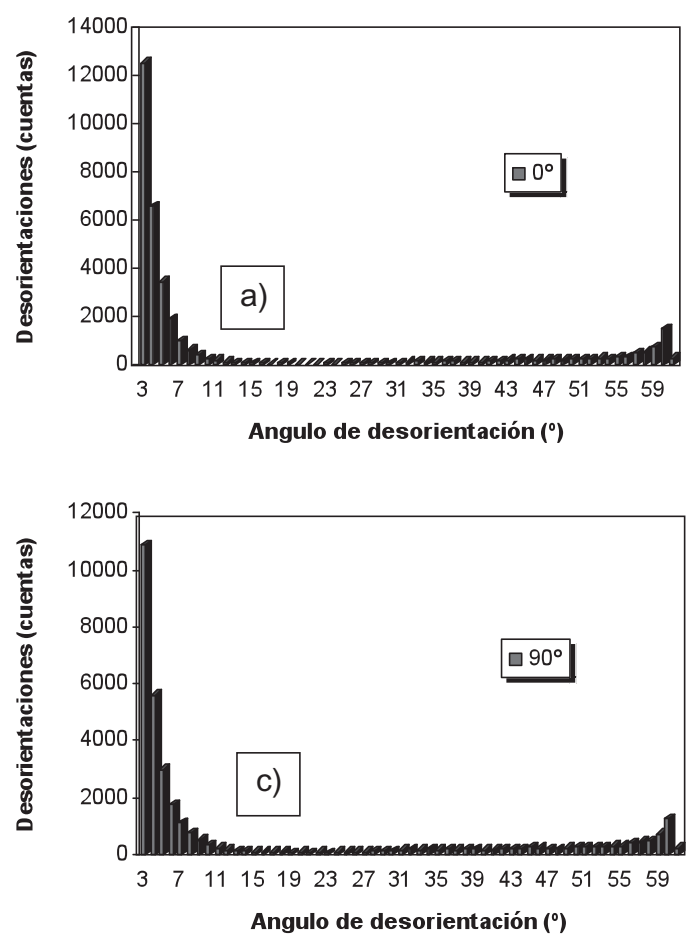

La laminación de la chapa produce la texturización del material con orientación de estructuras de mayor energía que favorecen la formación de subestructuras y subgranos con la deformación. Este hecho justificaría la influencia encontrada de la dirección de laminación sobre la distribución de desorientaciones de ángulo bajo.

\subsection{Desorientaciones en límite de grano}

El estudio de las desorientaciones de los límites de grano también se ha realizado teniendo en cuenta el modelo de las coincidence site lattice (CSLs), también llamadas fronteras especiales definidas por el parámetro å de acuerdo al criterio de Brandon ${ }^{[2]}$.

En la figura 13 aparecen representados los mapa de desorientaciones y CSLs del material de partida y una muestra con una deformación equivalente de 0,327. En la figura 13 a) se observan en rojo los límite de grano $\left(>15^{\circ}\right)$ y las maclas. En la $12 \mathrm{~b}$ ) se observa la distribución de las CSLs. En ellas se comprueba que las juntas $\sum 3$ representan una fracción muy importante de todas las juntas de ángulo alto, y
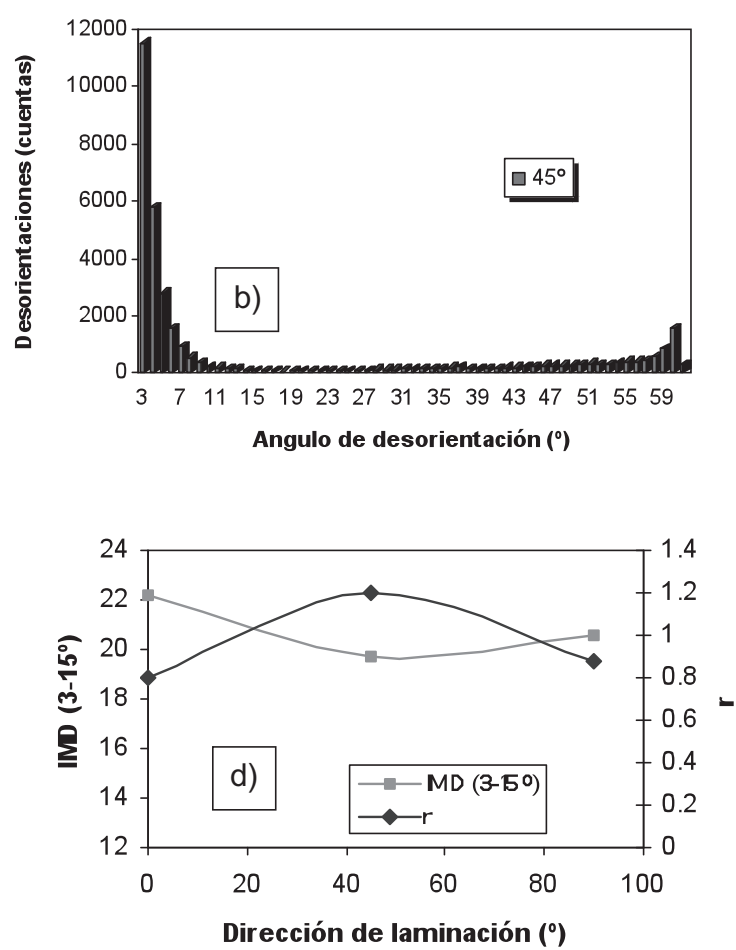

Figura 12. a), b) y c) Diagramas de desorientación de muestras deformadas a $0^{\circ}, 45^{\circ}$, y $90^{\circ}$, respecto a la dirección de laminación; d) Variación del IMD (3-15 $)$ y el índice de anisotropía con la dirección de laminación.

Figure 12. a), b) and c) Misorientation distribution histograms of deformed samples for $0^{\circ}, 45^{\circ}$ and $90^{\circ}$ respecting to rolling direction; d) IMD (3-15') and anisotropy indexes values as a function of rolling direction. 

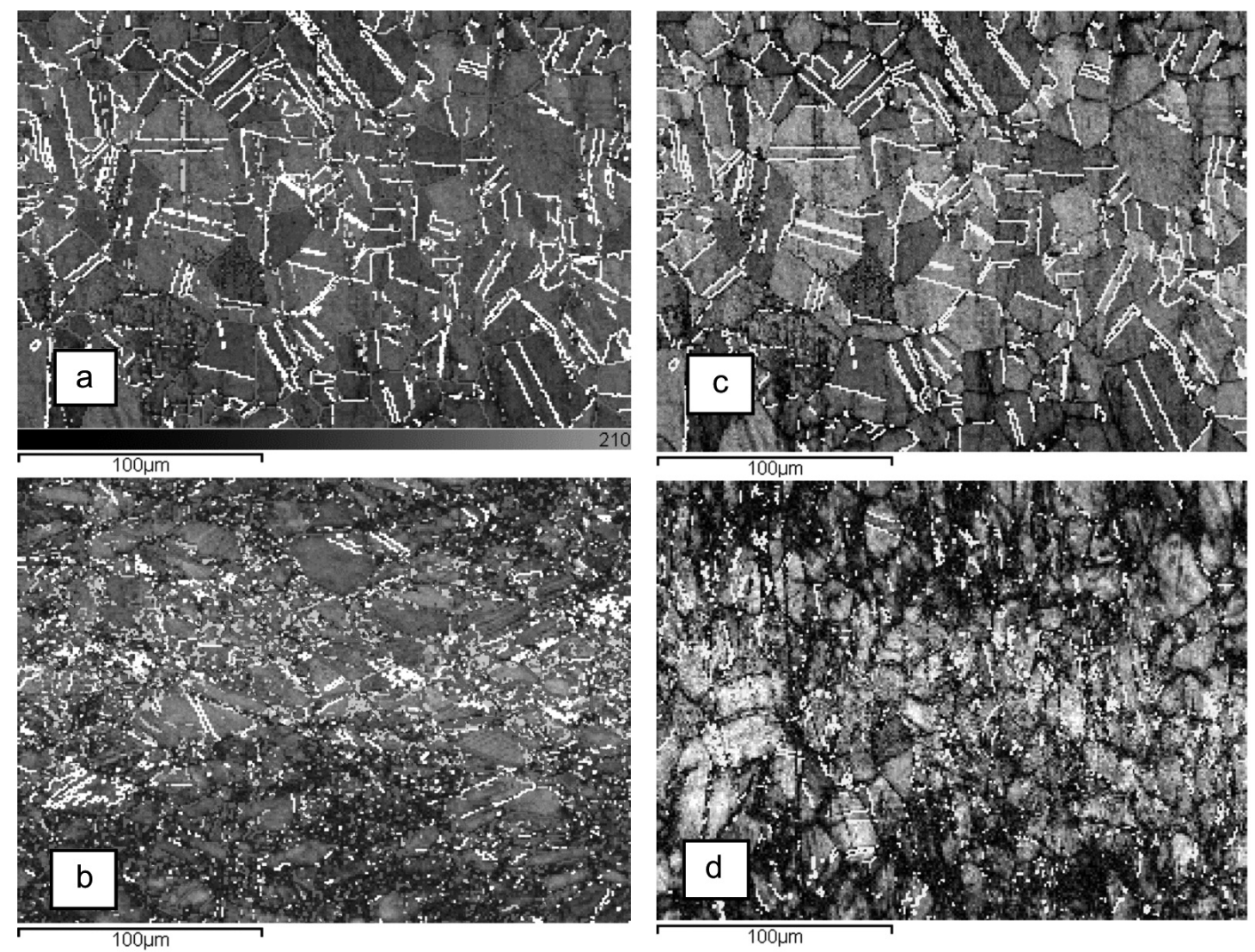

Figura 13. Mapas de desorientaciones del acero AISI 304: a) En estado de recepción; b) $\varepsilon_{\text {eq }} 0,327$. Mapas de CSLs del acero AISI 304; c) En estado de recepción; d) $\varepsilon_{\text {eq }} 0,327$.

Figure 13. Misorientation maps of AISI 304 steel. a)In initial conditions, b) $\varepsilon_{e q} 0.327$; CSLs maps of AISI $304 D D Q$ steel c) In initial condition d) $\varepsilon_{e q}: 0.327$.

coinciden con las maclas ya que las fronteras å3 son descritas como una rotación de $60^{\circ}$ sobre un eje $<111>$. La distribución de las diferentes CSLs obtenidas para cada una de las muestras aparece en la tabla III, en la que se demuestra la presencia mayoritaria de CSLs $\sum 3$.

Este efecto es analizado con más profundidad si se representa la distribución de las desorientaciones de ángulo alto para distintas deformaciones. En la figura 14 se observa una disminución de las desorientaciones de $60^{\circ}$ con la deformación. Si se consideran las fronteras $\Sigma 3$ como una rotación de unos $60^{\circ}$ sobre un eje $<111>$, la disminución de las $\Sigma 3$ justificaría el descenso del pico observado a los $60^{\circ}$ en la figura 14 en la que, conforme aumenta la deformación, la parte principal de la distribución permanece sin cambios pero el pico correspondiente a $\Sigma 3$ se ve fuertemente afectado. La intensidad del pico disminuye progresivamente conforme la deformación aumenta y el ángulo de rotación se desplaza a ángulos más bajos, produciendo un ensanchamiento del pico. Este ensanchamiento del pico nos da idea de la desviación del ángulo de rotación producido en cada caso con respecto al definido para las maclas en condiciones ideales $\left(60^{\circ}\right)$.

Mediante ambos análisis de desorientación, se demuestra que la distribución del carácter del límite de grano (GBCD) se ve modificada en gran medida en los procesos de deformación, observándose que, a bajas deformaciones se obtiene una GBCD que contiene una alta fracción de $\Sigma 3^{n}(n=1-3)$ y que la conexión de los límites de ángulo alto son interrumpidos por $\Sigma 9$ y $\Sigma 27$. Para deformaciones intermedias se obtiene una GBCD con una menor fracción de $\Sigma 3$ y una más alta fracción de $\Sigma 9$ y $\Sigma 27$, donde la conexión de las fronteras de ángulo alto no es interrumpida por las CSLs. Las desorientaciones a $60^{\circ}$ son típicas de la formación de maclas, pero las estructuras cristalinas cúbicas centradas en caras, como corresponde al acero ensayado, no suelen presentar la formación de maclas como mecanismo de deformación ${ }^{[2]}$. Por tanto, la disminución de las desorientaciones de ángulo alto se justifica por la variación con la deformación de juntas $\sum 3$. 
CARACTERIZACIÓN MEDIANTE LA TÉCNICA EBSD DE LA DEFORMACIÓN DE CHAPA DE ACERO INOXIDABLE AISI 304 DDQ BAJO TENSIONES MULTIAXIALES TÍPICAS DE LA EMBUTICIÓN CHARACTERIZATION BY EBSD TECHNIQUE OF AISI 304 DDQ STAINLESS STEEL SHEET DEFORMED UNDER TYPICAL DEEP DRAWING MULTIAXIAL STRESS SYSTEM

Tabla III. \% de las CSL con $\Sigma$ 3-45

Tabla III. CSLs content (\%) with $\Sigma$ 3-45

\begin{tabular}{|c|c|c|c|c|c|c|c|c|}
\hline \multirow{3}{*}{$\Sigma \mathrm{CSL}$} & \multicolumn{5}{|c|}{304 DDQ } & \multicolumn{3}{|c|}{$304 \mathrm{DDQ} \varepsilon_{\mathrm{eq}}: 0,065 \mathrm{~T}$} \\
\hline & - & $\varepsilon_{\mathrm{eq}}: 0,32$ & $\varepsilon_{\mathrm{eq}}: 0,32$ & $\varepsilon_{\mathrm{eq}}: 0,32$ & $\varepsilon_{\mathrm{eq}}: 0,14$ & - & $\varepsilon_{\mathrm{eq}}: 0,14$ & $\varepsilon_{\text {eq }}: 0,32$ \\
\hline & $0^{\circ}$ & $0^{\circ}$ & $45^{\circ}$ & $90^{\circ}$ & $0^{\circ}$ & $0^{\circ}$ & $0^{\circ}$ & $0^{\circ}$ \\
\hline 3 & 85,18 & 63,74 & 61,70 & 58,93 & 69,66 & 85,87 & 77,29 & 57,68 \\
\hline 5 & 0,59 & 1,85 & 2,06 & 2,03 & 1,12 & 0,25 & 0,72 & 2,82 \\
\hline 7 & 0,89 & 1,71 & 1,87 & 1,46 & 1,09 & 0,74 & 1,08 & 1,66 \\
\hline 9 & 1,79 & 3,62 & 4,17 & 4,33 & 2,76 & 1,89 & 1,64 & 4,41 \\
\hline 11 & 1,53 & 3,16 & 3,55 & 4,93 & 2,70 & 0,74 & 2,45 & 4,10 \\
\hline 13 & 1,04 & 1,99 & 1,65 & 1,82 & 1,84 & 0,55 & 1,01 & 2,31 \\
\hline 15 & 0,59 & 1,03 & 1,00 & 1,25 & 0,72 & 0,39 & 0,56 & 1,72 \\
\hline 17 & 0,89 & 2,97 & 1,98 & 3,16 & 4,87 & 1,13 & 2,79 & 2,50 \\
\hline 19 & 0,72 & 1,61 & 2,22 & 2,63 & 0,89 & 0,83 & 0,67 & 2,03 \\
\hline 21 & 0,68 & 1,58 & 1,46 & 1,31 & 0,72 & 0,58 & 0,70 & 1,25 \\
\hline 23 & 0,15 & 0,57 & 0,33 & 0,66 & 0,30 & 0,09 & 0,11 & 0,84 \\
\hline 25 & 0,68 & 1,90 & 2,36 & 1,25 & 1,25 & 1,13 & 1,21 & 1,75 \\
\hline 27 & 0,76 & 1,55 & 1,57 & 1,52 & 0,89 & 0,69 & 0,49 & 1,66 \\
\hline 29 & 0,51 & 1,39 & 1,57 & 1,85 & 1,02 & 0,76 & 0,94 & 2,22 \\
\hline 31 & 0,32 & 1,20 & 0,95 & 0,84 & 0,82 & 0,39 & 0,76 & 1,06 \\
\hline 33 & 0,57 & 1,90 & 2,38 & 2,27 & 1,45 & 0,25 & 0,97 & 1,94 \\
\hline 35 & 0,76 & 1,74 & 1,84 & 1,73 & 0,92 & 0,46 & 0,63 & 2,03 \\
\hline 37 & 0,21 & 0,82 & 1,00 & 0,78 & 0,63 & 0,30 & 0,56 & 1,13 \\
\hline 39 & 0,25 & 1,09 & 1,03 & 0,93 & 0,95 & 1,08 & 0,49 & 1,41 \\
\hline 41 & 0,34 & 1,58 & 2,06 & 2,18 & 1,12 & 0,60 & 0,81 & 2,28 \\
\hline 43 & 0,96 & 1,82 & 2,00 & 2,39 & 2,70 & 0,78 & 2,68 & 1,60 \\
\hline 45 & 0,62 & 1,20 & 1,25 & 1,76 & 1,58 & 0,51 & 1,42 & 1,60 \\
\hline
\end{tabular}

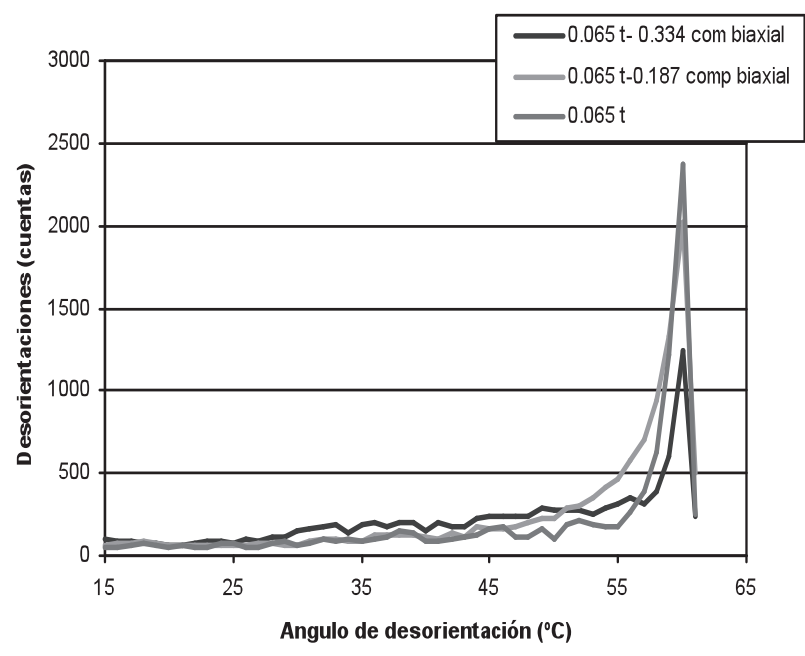

Figura 14. Variación de las desorientaciones de ángulo alto con la deformación.

Figure 14. High angle misorientations values as a function of deformation grade.

\section{CONCLUSIONES}

La acritud inicial practicada sobre el material presenta una correlación directa con el endurecimiento final obtenido tras su deformación en condiciones de tracción-compresión biaxial, similar a la que se produce en los procesos de embutición de chapa.

Se ha caracterizado la microtextura del acero AISI 304 DDQ mediante la técnica EBSD, justificando que la resolución del método es adecuada para el análisis de la zona de deformación homogénea tras el ensayo de estirado en condiciones multiaxiales típicas del proceso de embutición. Con dicha técnica se ha establecido una correlación directa entre la formación de subestructuras y la deformación en condiciones T-CC.

La comparación entre las texturas de las muestras en estado de recepción y con acritud inicial revela que el gradiente de desorientaciones intragranulares producidas en la deformación no depende significativamente de las condiciones iniciales del material. 
Las desorientaciones de ángulo bajo obtenidas en muestras deformadas a $0^{\circ}, 45^{\circ}$ y $90^{\circ}$, respecto a la dirección de laminación del material, presentan una correlación inversa con la anisotropía normal del material. La dirección con menor tendencia al adelgazamiento se corresponde con la que presenta un menor número de desorientaciones de ángulo bajo.

El análisis de desorientaciones de ángulo alto $\left(>15^{\circ}\right)$ revela la existencia de la variación de la distribución del carácter del límite de grano con la deformación. Particularmente la fracción de juntas $\sum 3$ disminuye con la deformación, lo que justifica la disminución de desorientaciones a $60^{\circ}$ encontradas y que no se pueden justificar con mecanismos de deformación transcristalinos.

El análisis efectuado mediante la técnica EBSD demuestra la validez de la misma como método de análisis de la embutibilidad del acero AISI 304 DDQ, dadas las correlaciones encontradas entre el proceso de deformación con tensiones típicas de la embutición y el efecto producido en la microtextura de la chapa.

\section{REFERENCIAS}

[1] G. Berming, J. Tobisch y K. Helming, Proc. of the $10^{\text {th }}$ Int. Conf. Textures of Materials,1994, Vol. 157-162, pp. 1.953-1.961.

[2] V. Randle, Introduction to Texture Analysis: Macrotexture, Microtexture and Orientation Mapping, Ed. OPA, Florida, 2000, cap 6, pp. 127-142.

[3] A.J. Schwartz et al., Electron Backscattered Diffraction in Materials Science, Ed. Plenum Publishers, New York, 2000, cap. 19, pp. 231-244.

[4] M. El Wahabi, L. Gavard, F. Montheillet, J.M. Cabrera y J.M. Prado, Acta Mater. 53 (2005) 4605-4612.

[5] D. Jorge-Badiola, A. Iza-Mendia e I. Gutiérrez, Mater. Sci. Eng. A 394 (2005) 445-454.

[6] M. El Wahabi, L. Gavard, J.M. Cabrera, J.M. Prado y F. Montheillet, Mater. Sci. Eng. A 393 (2005) 83-90.

[7] M. El Wahabi, J. M. Cabrera y J. M. Prado, Mater. Sci. Eng. A 343 (2003) 116-125.

[8] Q. Xue, J.F. Bingert, B.L. Henrie y G.T. Gray, Mater. Sci. Eng. A 473 (2008) 279-289.

[9] Q. Xue, X.Z. Liao, Y.T. Zhu y G.T. Gray, Mater. Sci. Eng. A 410-411 (2005) 252-256.

[10] Q. Xue, M.A. Meyers y V.F. Nesterenko, Mater. Sci. Eng. A 384 (2004) 35-46.
[11] M. A. Meyers, Y.B. Xu, Q. Xue, M.T. PérezPrado y T.R. McNelley, Acta Mater. 51 (2003) 1.307-1.325.

[12] M. Humbert, B. Petit, B. Bolle y N. Gey, Mater. Sci. Eng. A 454-455 (2007) 508-517.

[13] B. Petit, N. Gey, M. Cherkaoui, B. Bolle y M. Humbert, Int J Plasticity 23 (2007) 323-341.

[14] V. Miguel, Tesis Doctoral, Universidad Politécnica de Valencia, 2005.

[15] V. Miguel, J. Coello, A. Calatayud, C. Ferrer y A. Martínez. Proc. Int. Deep Drawing Research Group, 2007, pp. 221-228.

[16] UNE EN ISO 10088-2: Parte 2.

[17] V. Miguel, C. Ferrer y A. Calatayud. Rev. Metal. Madrid 43 (2007) 142-152.

[18] V. Miguel, C. Ferrer, A. Calatayud, A. Martínez y J. Coello, Proc. Int.Deep Drawing Research Group, IDDRG'06, 2006, pp. 223-229.

[19] V. Miguel, J. Benet, J. Coello, A. Calatayud y A. Martínez, Materials Processing and Design. Modeling, Simulation and Applications, 2007, pp. 823-828.

[20] M. Kamaya, A.J. Wilkinson y J.M. Titchmarsh, Acta Mater. 54 (2006) 539-548.

\section{Nomenclatura}

T-CC Tracción compresión biaxial

$\mathrm{h}_{\mathrm{o}} \quad$ Anchura inicial de la probeta de ensayo

$\mathrm{h} \quad$ Anchura final de la probeta tras el ensayo

1. Diámetro inicial de marcas

$l_{1} \quad$ Dimensión longitudinal de las marcas tras el ensayo

$\mathrm{L}_{\mathrm{t}} \quad$ Dimensión transversal de las marcas tras el ensayo

$\varepsilon_{t} \quad$ Deformación transversal

$\varepsilon_{1} \quad$ Deformación longitudinal

$\varepsilon_{z} \quad$ Deformación en espesor

$\varepsilon_{\text {eq }} \quad$ Deformación equivalente de Von Misses en T-CC

$\varepsilon_{\text {eqo }} \quad$ Deformación equivalente de Von Misses inicial

IMD Densidad de desorientaciones

$\phi_{1}, \phi_{\mathrm{o}}, \phi_{\max }$ Angulo de desorientación en cada punto, mínimo y máximo

N Número de pixels del área escaneada.

CSL Frontera de geometría especial (coincident sites lattices)

¿n Tipo de frontera de geometría especial, n: $1-45$

GBCD Distribución del carácter del límite de grano 